\title{
Editorial: Complications of Neck Dissection for Thyroid Cancer
}

\author{
Ashok R. Shaha, MD FACS ${ }^{1,2}$ \\ ${ }^{1}$ Attending Surgeon, Memorial Sloan-Kettering Cancer Center, Head and Neck Service, 1275 York Avenue, New York, NY 10065, \\ USA \\ ${ }^{2}$ Professor of Surgery, Cornell University Medical Center, New York, New York, USA
}

There is a high incidence of nodal metastasis in well-differentiated and medullary thyroid carcinomas. With a diagnosis of papillary carcinoma, $40 \%$ to $60 \%$ will develop nodal metastasis. ${ }^{1}$ Elective nodal dissection is generally not indicated, however careful evaluation of the central compartment is essential in all patients undergoing thyroid surgery for suspected thyroid cancer. ${ }^{2}$ If the lymph nodes are clinically apparent at the time of thyroidectomy, central compartment clearance is usually considered. Neck dissection, though a reliably safe procedure, carries certain potential complications which are important to recognize. Roh et al. ${ }^{3}$ from Korea have mainly discussed the issue related to chyle leak after neck dissection for thyroid cancer. The purpose of this editorial is to discuss various types of neck dissections for thyroid cancer and routine complications, along with the techniques to avoid these complications.

The classical radical dissection was described in 1906 by George Crile, who was the first surgeon to describe in systematic detail the surgical procedure of radical neck dissection, which included removal of all the lymph nodes in the neck, along with three important structures - the sternomastoid muscle, the internal jugular vein, and the accessory nerve. The major drawback to this surgical procedure was shoulder dysfunction due to sacrifice of the accessory

Received October 31, 2007; accepted November 1, 2007; published online: December 6, 2007.

Address correspondence and reprint requests to: Ashok R. Shaha, MD FACS; E-mail: . shahaa@mskcc.org

Published by Springer Science+Business Media, LLC $\odot 2008$ The Society of Surgical Oncology, Inc. nerve. In 1962 Oswaldo Suarez, an Argentinian surgeon, described the facial compartments in the neck and the facial envelope covering a selective group of lymph nodes. He discussed a modification of radical neck dissection, which he described as a functional neck dissection. The philosophy behind this surgical procedure was to remove a selected group of lymph nodes and to preserve the vital structures, including the accessory nerve, jugular vein, and sternomastoid muscle. This operation was popularized by Ettore Bocca and Caesar Gavilan in Europe, and in the United States by surgeons from MD Anderson Hospital: Richard Jesse, Alando Ballantyne, and Robert Byers.

As the biology of metastatic neck disease became clearer and the patterns of nodal metastasis were well defined, there was growing emphasis on modified neck dissection. The major pendulum swing from radical neck dissection to modified neck dissection occurred in the early 1980s. Today, the radical neck dissection is performed only in select circumstances where extensive metastatic disease exists in the neck and the accessory nerve cannot be preserved. For better functional outcome, every effort is now made to preserve the accessory nerve if disease is not encircling the nerve.

Even though the incidence of nodal metastasis is quite high in thyroid cancer, it has very little prognostic implication. Several published series failed to show a major impact of nodal metastasis in long-term outcome. However, nodal metastasis does have some impact in elderly individuals or in patients with bulky nodal disease. ${ }^{4-6}$ 
There is considerable debate regarding elective nodal dissection in patients with well-differentiated thyroid carcinoma. Routine paratracheal clearance is not indicated in all such patients. However, routine evaluation of the paratracheal area is recommended to check for any obvious suspicious lymph nodes. If a paratracheal-area lymph node is suspicious, a frozen section may be obtained. Appropriate paratracheal clearance is recommended based on the pathological finding. There is a high incidence of temporary hypoparathyroidism in patients undergoing central compartment clearance. If, at the time of surgery, there is obvious metastatic disease in the jugular chain or lateral neck, modified neck dissection is generally recommended.

Berry picking is a procedure which was used in the 1960s and 1970s. The philosophy behind berry picking was to remove only suspicious and enlarged lymph nodes, as lymph node removal has no major impact on long-term outcome. The biology of metastatic thyroid cancer is very different from that of squamous cell carcinoma, and the berry picking procedure became quite popular. However, this operation did not appear to be biologically sound, as there would invariably be other lymph nodes harboring metastatic thyroid carcinoma. There was a high incidence of recurrent disease in the neck, requiring another surgical procedure. Even though sentinel node biopsy has been attempted in thyroid cancer, I do believe there is no clinical application of this technique in the management of well-differentiated thyroid cancer.

The current surgical procedure is modified neck dissection with removal of the lymph nodes at levels II, III, IV, and V. The incidence of nodal metastasis in thyroid carcinoma at level I (submandibular lymph nodes) is quite rare, and level I dissection is not routinely indicated for fear of injury to the ramus mandibularis, resulting in lip weakness. The incidence of nodal metastasis is also quite low at level II $b$ and $\mathrm{Va}$, and these regions may not need routine dissection unless there is obvious disease.

Modified neck dissection for thyroid carcinoma preserves all the important structures, such as the sternomastoid muscle, internal jugular vein, and accessory nerve, along with the submandibular salivary gland. Paratracheal clearance should include removal of the lymph nodes, both at levels VI and VII, if they are clinically apparent. Level VI lymph nodes include paratracheal nodes, while level VII includes superior mediastinal nodes, which are more of concern in patients with medullary thyroid carcinoma or patients with obvious metastatic disease at level VI. There is growing interest in selective neck dissection in thyroid cancer where compartmentbased neck dissection is performed for a select group and levels of nodes.

The incision for total thyroidectomy and modified neck dissection includes a transverse incision in the suprasternal area, with a J-shaped or a hockey stick incision up to the mastoid process. Most surgeons now use a curvilinear or a horizontal incision at the level of the cricoid cartilage, which encompasses both the surgical procedures of total thyroidectomy and modified neck dissection with one incision. A McFee incision (double transverse incision) in the neck is not recommended due to cosmetic considerations.

Even though modified neck dissection is a reliable, safe surgical procedure for metastatic thyroid carcinoma, due consideration must be given to certain complications. ${ }^{7}$ These include: high incidence of temporary and permanent hypoparathyroidism, recurrent laryngeal nerve injury, accessory nerve weakness leading to shoulder disability, chyle leak, bleeding and hematoma, Horner's syndrome, and injury to the brachial plexus or hypoglossal nerve (Table 1). Total thyroidectomy and neck dissection include considerable dissection in the paratracheal area, especially along the recurrent laryngeal nerve. This has a direct implication in a higher incidence of nerve injury and injury to the parathyroid glands, leading to temporary or permanent hypoparathyroidism. The patient undergoing neck dissection should be observed carefully in the postoperative period for serum calcium levels and symptoms of hypocalcemia, such as carpopedal spasm or tetany. These patients should be treated and maintained on calcium and vitamin D supplementation until the serum calcium returns to normal levels, which may take two to three months.

At the time of surgery, if any portion of the parathyroid gland is identified to be devascularized, it should be autotransplanted, preferably in the sternomastoid muscle or in the strap muscles. For a total thyroidectomy, there is no need to autotransplant a parathyroid gland in the forearm. Prior to any autotransplantation of the parathyroid gland, it is vitally important to send a small piece for frozen section to confirm that the autotransplanted tissue is truly a parathyroid gland, and not a lymph node or metastatic thyroid carcinoma. The parathyroid tissue is minced into minute pieces, and placed in the interstices of the sternomastoid muscle. Bilateral recurrent laryngeal nerve palsy is quite rare. However, patients undergoing bilateral modified neck dissection should be observed very closely for this 
TABLE 1. Complications of neck dissection for thyroid cancer

\begin{tabular}{l}
\hline Central neck dissection \\
\hline 1) Hypoparathyroidism - temporary/permanent \\
2) Recurrent laryngeal nerve injury \\
3) Superior laryngeal nerve injury \\
Lateral neck dissection \\
1) Hypoparathyroidism - temporary/permanent \\
2) Chyle leak \\
3) Hemorrhage \\
4) Seroma \\
5) Wound infection \\
6) Nerve injuries - accessory, ramus mandibularis, sympathetic \\
(Horner's syndrome), phrenic, brachial plexus, cutaneous \\
cervical plexus
\end{tabular}

complication. If there is any airway issue in the recovery room, the patient should be monitored closely, evaluated with fiber-optic laryngoscopy, and reintubated. True bilateral vocal cord paralysis may require tracheostomy to secure the airway.

The thoracic duct, which runs into the posterior mediastinum, generally opens up at the junction of the left jugular vein and subclavian vein. There is a high incidence of nodal metastasis at level IV in thyroid carcinoma and injury to the lymphatic structures is quite common, leading to chyle leak. If a chyle leak is identified at the time of surgery, as Roh et al. ${ }^{3}$ have mentioned, it should be corrected by ligation of nonabsorbable ties, silk or prolene, or with hemoclips. If there is a continuous leak in this region, transfixion stitches with silk or prolene should be placed, with muscle buttress from the sternomastoid. Most of the time, chyle leaks can be controlled in the operating room. However, it is not uncommon, in spite of good control on the operating table, to see a postoperative chyle leak within the first 24 hours or after a few days.

The majority of patients with total thyroidectomy and neck dissection require placement of a drain. We generally use a closed suction drain, such as Reliavac or Jackson Pratt. A major chyle leak in the postoperative period is easily identifiable, with a large amount of chylous fluid flowing into the drainage system. A conservative approach with observation, antibiotics, pressure dressing, and a fat-free (medium-chain triglyceride) diet successfully heals almost $90 \%$ of the patients. However, a small number of patients will have an excessive amount of drainage. If the drainage is more than $500 \mathrm{cc}$ per day for an extended period of time, due consideration may be given to surgical exploration, identification of the chyle leak, and suturing. This may be quite a difficult procedure, as the tissues have become inflamed and any placement of stitches leads to further laceration of the soft tissue. Under these circumstances, a biological sealant, such as Flo-seal, may be used. A chyle leak in the wound leads to chemical inflammatory reaction in the skin and subcutaneous tissue, which needs to be observed closely. Occasionally, the patient may end up with a chyloma, which can be easily aspirated percutaneously. Multiple aspirations may be required to resolve a chyloma. Some investigators have tried injection of tetracycline or other sclerosing agents in the neck. However, this may lead to considerable scarring and fibrosis, inflammatory reaction, and pain.

Seroma is a fairly common complication after total thyroidectomy and neck dissection, which invariably can be treated conservatively, either by observation or repeated aspirations. Most seromas will resolve in a few days. Wound infection is quite rare. ${ }^{7}$ If the wound is edematous or fluctuant, it may necessitate aspiration or opening the wound and packing.

The complications related to Horner's syndrome (injury to sympathetic trunk), and brachial plexus or, hypoglossal nerve are quite rare. $^{7}$ The accessory nerve may be injured by extensive dissection and skeletization of the nerve with neuropraxia and devascularization. Most of the time, shoulder function can improve with intensive physiotherapy.

Understanding of the patterns and biology of metastatic thyroid cancer along with the technical details of neck dissections and complications are crucial for safe recovery from neck dissection in patients with thyroid cancer.

\section{REFERENCES}

1. Hundahl SA, Fleming ID, Fremgen AM, et al. A National Cancer Data Base report on 53,856 cases of thyroid carcinoma treated in the U.S., 1985-1995. Cancer 1998; 83:2638-48.

2. Shaha AR. Management of the neck in thyroid cancer. Otolaryngol Clin North Am 1998; 31:823-31.

3. Roh et al. Prospective Identification of Chyle Leakage in Patients Undergoing Lateral Neck Dissection for Metastatic Thyroid Cancer (ASO-2007-08-0602).

4. Sugitani I, Kasai N, Fujimoto Y, et al. A novel classification system for patients with PTC: addition of the new variables of large $(3 \mathrm{~cm}$ or greater) nodal metastases and reclassification during the follow-up period. Surgery 2004; 135:139-48.

5. Noguchi S, Murakami N, Yamashita H, et al. Papillary thyroid carcinoma: modified radical neck dissection improves prognosis. Arch Surg 1998; 133:276-80.

6. Hughes CJ, Shaha AR, Shah JP, et al. Impact of lymph node metastasis in differentiated carcinoma of the thyroid: a matched-pair analysis. Head Neck 1996; 18:127-32.

7. Cheah WK, Arici C, Ituarte PH, et al. Complications of neck dissection for thyroid cancer. World J Surg 2002; 26:1013-6. 